\title{
CYCS Gene
}

National Cancer Institute

\section{Source}

National Cancer Institute. CYCS Gene. NCI Thesaurus. Code C129849.

This gene is involved in electron transport. 